\title{
Costo del proceso diagnóstico del cáncer de mama en las Unidades de Medicina Familiar del Instituto Mexicano del Seguro Social, Querétaro, México
}

\author{
Verónica Escorcia-Reyes 1,2, Enrique Villarreal-Ríos ${ }_{1}^{1}$, Emma Rosa Vargas-Daza1, \\ Liliana Galicia-Rodriguez ', 'Lidia Martínez-González 1', Eduardo Paredes-Hernández3, \\ Silvia Isabel Ayala-García. ${ }^{3}$ \\ ${ }^{1}$ Unidad de Investigación Epidemiológica y en Servicios de Salud, Querétaro; ${ }^{2}$ Unidad de Medicina Familiar No. 16 \\ "Dr. Arturo Guerrero Ortiz", Delegación Querétaro; ${ }^{3}$ Hospital General Regional No.1, Delegación Querétaro. Instituto \\ Mexicano del Seguro Social.
}

\section{RESUMEN}

Objetivo: Determinar el costo del proceso diagnóstico del cáncer de mama. Métodos: Estudio de costos, en mujeres de 40 años y más, de enero a diciembre del 2010 de las unidades de medicina familiar del Instituto Mexicano del Seguro Social. La muestra se calculó con la fórmula de promedios para población infinita para un total de 245 mujeres. El costo se estimó mediante la técnica de tiempos y movimientos y consulta de expertos; el costo por uso de intervención se calculó con el uso promedio por intervención multiplicado por el costo unitario del mismo. El costo total del proceso diagnóstico se obtuvo de la suma de los costos de cada uno de las intervenciones. El plan de análisis incluyó promedios, porcentajes e intervalos de confianza. Resultados: El costo promedio del proceso diagnóstico fue de $\$ 852,45$. Conclusión: El proceso diagnóstico de cáncer de mama incluye tamizaje y casos confirmados. El costo del proceso diagnóstico es relativamente bajo ofreciendo mejores perspectivas para el paciente y el sistema de salud.

\section{PALABRAS CLAVE. Cáncer de mama, proceso diagnóstico, costo}

\section{SUMMARY}

Objective: To determine the cost of the diagnostic process for breast cancer. Methods: Costs were calculated, in women 40 years and older, from January to December 2010 of the Family Medicine Units of the Mexican Social Security Institute. The sample was calculated with the formula for infinite population averages for a total of 245 women. The cost was estimated by time and motion technique and expert consultation, the intervention cost was calculated using the average usage per intervention multiplied by the unit cost thereof. The total cost of the diagnostic process was obtained from the sum of the costs of each of the interventions. The analysis plan included means, percentages and confidence interval. Results: The average cost of the diagnostic process was $\$ 852.45$. Conclusions: The diagnostic process for breast cancer include screening and confirmatory cases. The cost of the diagnostic process is low, offers better prospects for the patient and the health system.

KEY WORDS: Breast cancer, diagnostic process, cost 


\section{INTRODUCCIÓN}

Actualmente, el cáncer de mama es una de las principales causas de muerte en las mujeres, por lo que constituye una prioridad en salud. Representa un reto para el financiamiento y protección financiera del sistema de salud, en particular para los países en desarrollo (1).

Se ha observado que el diagnóstico de las pacientes se realiza en etapas muy avanzadas (2), lo que implica un mayor costo y utilización de servicios, de ahí la importancia de identificar esta patología en estadios tempranos que correspondan al periodo pre patogénico de la historia natural de la enfermedad (3).

Anteriormente el éxito del control de cáncer de mama recaía en el tratamiento (4), ahora, se basa en un adecuado proceso diagnóstico, que tiene como piedra fundamental el tamizaje o cribado, definido como la aplicación de procedimientos de selección que pueden ser empleados en población aparentemente sana con el objeto de identificar la enfermedad (5).

La detección temprana del cáncer de mama mediante el tamizaje con mastografía ha mostrado disminuir las tasas de mortalidad entre 28 a $65 \%$, con una media de $46 \%$ (6), sin embargo, el acceso y utilización de los servicios de salud para el diagnóstico de cáncer de mama en México son escasos, situación que también se aplica a los demás países latinoamericanos (1).

La planificación de los servicios de salud requiere un concepto de costos que va más allá de la idea de la cantidad de dinero que el Sistema Nacional de Salud paga para adquirir bienes o servicios (7).

Por costos se entiende el valor de los recursos para producir algo o la suma de erogaciones en que se incurre para la adquisición de un bien o de un servicio, con la intensión de que genere ingresos en el futuro. En el caso de los costos en salud, no solo se miden ingresos, sino también beneficios, bienestar y calidad de vida (7).

Estudios realizados sobre el costo de la atención del cáncer de mama en México, señalan que la detección temprana y oportuna es la alternativa más efectiva en términos del costo. El costo de atención promedio por año-paciente se estimó en $\$ 110.459$, sólo el $14 \%$ se diagnosticó en fase I y el $48 \%$ en las fases III-IV. En 2002, el costo del diag- nóstico en etapa I fue de $\$ 74.522$, comparado con $\$ 102.042$ en la etapa II, \$154.018 en la etapa III y $\$ 199.274$ en la etapa IV (8).

El costo de la fase diagnóstica se ha identificado en $\$ 347$, cuando se considera a todas las personas sometidas a un proceso de detección y evaluación por cáncer de mama, y de $\$ 68,887$ si se considera sólo a las pacientes cuyo diagnóstico final es cáncer de mama. Por lo cual el costo total del diagnóstico corresponde a \$7.233 (8).

En España, el costo por mujer que no precisa recribado es de $€ 30,30$ (\$516,97), a esta cantidad hay que añadir $€ 33,33(\$ 517,48)$ si la mujer necesita recribado mediante mamografía diagnóstica, $€ 45,45(\$ 775,45)$ si el recribado es mediante una ecografía y $€ 78,78(\$ 1.344,11)$ si el recribado es mediante ecografía más mastografía (9).

El objetivo del artículo es identificar el costo del proceso diagnóstico de cáncer de mama en México.

\section{MATERIAL Y MÉTODOS}

Se realizó un estudio de costos en mujeres de 40 años y más en el periodo de enero a diciembre de 2010 en las unidades de medicina familiar del Instituto Mexicano del Seguro Social en México, delegación Querétaro. Se incluyeron todas aquellas mujeres a las que se les realizó mastografía y cumplieron con el proceso diagnóstico establecido para detección de cáncer de mama.

El tamaño de la muestra se calculó con la fórmula de promedios para población infinita, con nivel de confianza del $95 \%$ para una cola $(Z=1.64)$, considerando que el costo promedio fue $\$ 1.000$, para un total de 245 pacientes. La técnica muestral fue aleatoria simple, utilizando como marco muestral el listado de pacientes a las que se les realizó la mastografía. Se analizaron variables sociodemográficas (edad, escolaridad, estado civil) y de salud (número de embarazos).

El proceso diagnóstico se valoró en función de (Figura 1):

a. Intervenciones (mastografía, ecografía, biopsia, consulta de medicina preventiva, medicina familiar, clínica de mama).

b. Uso por intervención (número de veces que fue utilizado el servicio o la intervención durante el proceso diagnóstico para cáncer de mama).

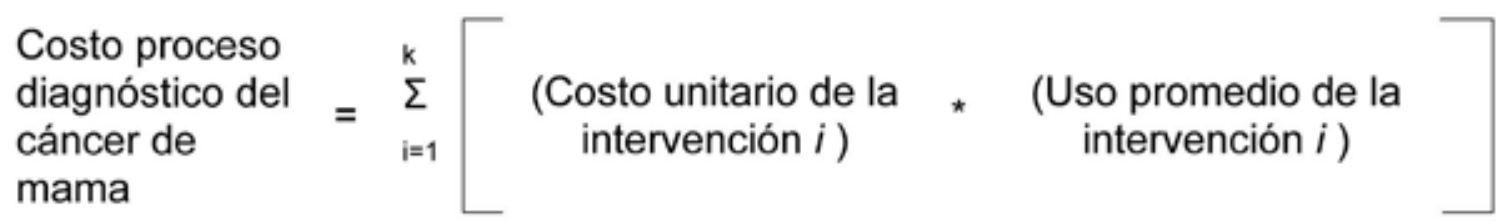

Figura 1. Fórmula para el cálculo del costo del proceso diagnóstico del cáncer de mama. 
c. Costo unitario (se estimó mediante la técnica de tiempos y movimientos [costos fijos] y consulta de expertos [costo variable]).

d. Costo promedio por intervención (se calculó multiplicando el uso por intervención por el costo unitario del mismo).

e. Costo total del proceso diagnóstico (se obtuvo de la suma de los costos promedio de cada uno de las intervenciones).

Se consideró el resultado de la mastografía de acuerdo a la clasificación del sistema BI-RADS y el resultado de la biopsia para el diagnóstico definitivo de cáncer de mama.

El procedimiento para la recolección de la información se inició en el servicio de mastografía, identificando los registros de las mastografías realizadas en el periodo comprendido. Se obtuvo un registro de las pacientes, a partir del cual se acudió al expediente electrónico y clínico para identificar el uso de los servicios de medicina preventiva, medicina familiar, laboratorio, gabinete y clínica de mama. Cuando la paciente fue referida a clínica de mama se buscó el uso todos aquellos otros servicios solicitados por el médico tratante, incluyendo otro estudio de mastografía para corroborar diagnóstico, ecografía, biopsia, valoración preoperatoria y otros estudios de imagen.

El plan de análisis incluyó promedios, porcentaje e intervalos de confianza. A partir del costo promedio total se realizó proyección del costo para una población específica. También se incluyó como parte del análisis la identificación de los posibles escenarios diagnóstico los cuales se construyeron en base al comportamiento que se sigue en las diferentes etapas del proceso diagnóstico.

Para mejor compresión de las cifras, las equivalencias de \$1 (peso mexicano) en US\$ (dólar estadounidense) y $€$ (euro) al $1^{\circ}$ de enero de 2012 fue de 0,0717 y 0,0560 respectivamente.

\section{RESULTADOS}

El promedio de edad de la población estudiada fue 53,5 años (IC 95\%: 52,53-54,48 años), la vida en pareja está presente en 73,9\% (IC 95\%: 68,4$79,4 \%$ ), la escolaridad predominante es primaria o menos en $60,8 \%$ (IC 95\%: $54,7-66,9 \%$ ) y el promedio de embarazos fue 4,18 (IC 95\%: 3,88-4,48 embarazos).

El total de mastografías realizadas reportó 86,5\% (IC 95\%: 82,2-90,8\%) sin sospecha de cáncer de mama (BI-RADS 0,1 y 2). Al 5,7\% de pacientes se les realizó una segunda mastografía, de estas el 57,1\% (IC 95\%: 50,9-63,3\%) se reportó sin sospecha de cáncer de mama (BI-RADS 0 y 2). En la Tabla I se presentan estos resultados en base a la clasificación BI-RADS.
Tabla I

\section{RESULTADO DE LA PRIMERA Y SEGUNDA MASTOGRAFÍA EN EL PROCESO DIAGNÓSTICO DEL CÁNCER DE MAMA}

\begin{tabular}{lccc}
\hline \multirow{2}{*}{ Clasificación } & Porcentaje & \multicolumn{2}{c}{ IC 95\% } \\
& & & \\
& & & \\
Inferior & Superior \\
Primera mastografía & 6,1 & 3,1 & 9,1 \\
BI-RADS 0 & 3,3 & 1,0 & 5,5 \\
BI-RADS 1 & 77,1 & 71,9 & 82,4 \\
BI-RADS 2 & 9,4 & 5,7 & 13,0 \\
BI-RADS 3 & 3,7 & 1,3 & 6,0 \\
BI-RADS 4 & 0,4 & 0,0 & 1,2 \\
BI-RADS 5 & & & \\
Segunda mastografía & 7,1 & 3,9 & 10,4 \\
BI-RADS 0 & 50,0 & 43,7 & 56,3 \\
BI-RADS 2 & 14,3 & 9,9 & 18,7 \\
BI-RADS 3 & 7,1 & 3,9 & 10,4 \\
BI-RADS 4 & 21,4 & 16,3 & 26,6 \\
BI-RADS 5 & & & \\
\hline
\end{tabular}

La prevalencia de cáncer de mama confirmada mediante biopsia fue de 2,4\% (IC 95\%: 0,5-4,3\%). Del total de biopsias realizadas el $31,3 \%$ (IC 95\%: 25,5-37,1\%) corresponde a biopsia por aspiración de aguja fina, el 43,8\% (IC 95\%; 37,6-50,0\%) corresponde a biopsia excisional y el 25,0\% (IC 95\%; 19,6-30,4) a biopsia con marcaje.

El servicio con más alta utilización fue medicina preventiva, el promedio por paciente fue 1,649 atenciones, seguido de la mastografía con 1,057 atenciones. En la Tabla II se presenta el uso promedio por tipo de servicio a lo largo de todo el procedimiento diagnóstico de cáncer de mama.

El costo unitario más alto corresponde a biopsia con $\$ 1.635,14$ y el costo por servicio ajustado por uso promedio más alto es la mastografía con $\$ 390,04$. El costo promedio del proceso diagnóstico de cáncer de mama aplicado a una población de mujeres de 40 años y más es $\$ 852,46$. En la Tabla II se presentan los costos unitarios y los costos por servicio.

Cuando se proyecta (Tabla III) el costo promedio del proceso diagnóstico de cáncer de mama a una población específica, se identifica que el gasto necesario para una población de 100.000 mujeres es ochenta y cinco mil millones de pesos (\$85.000.000.000).

Cuando se analizan los posibles escenarios diagnósticos se identifica que el de mayor costo corresponde a la biopsia marcaje con arpón el cual reporta un costo de $\$ 5 \cdot 160,11$. En la Tabla IV se presenta el costo para cada escenario. 
Tabla II

USO PROMEDIO, COSTO UNITARIO, COSTO PROMEDIO Y COSTO TOTAL DE LOS SERVICIOS EMPLEADOS EN EL PROCESO DIAGNÓSTICO DE CÁNCER DE MAMA

\begin{tabular}{|c|c|c|c|}
\hline & Uso & Costo unitario* & Costo promedio* \\
\hline \multicolumn{4}{|l|}{ Intervenciones } \\
\hline Mastografía & 1,057 & 368,95 & 390,04 \\
\hline Ecografía & 0,073 & 320,95 & 23,58 \\
\hline Biopsia & 0,065 & $1.635,14$ & 106,78 \\
\hline Consulta de medicina preventiva & 1,649 & 95,57 & 157,59 \\
\hline Consulta de médico familiar & 0,257 & 133,96 & 34,45 \\
\hline Consulta de clínica de mama & 0,273 & 179,04 & 48,96 \\
\hline Valoración preoperatoria & 0,057 & 174,04 & 9,94 \\
\hline \multicolumn{4}{|l|}{ Estudios de laboratorio } \\
\hline Biometría hemática & 0,110 & 60,00 & 6,61 \\
\hline Glucosa & 0,110 & 20,00 & 2,20 \\
\hline Tiempo parcial de tromboplastina & 0,094 & 20,00 & 1,88 \\
\hline Acido úrico & 0,102 & 30,00 & 3,06 \\
\hline Urea & 0,102 & 20,00 & 2,04 \\
\hline Creatinina & 0,102 & 20,00 & 2,04 \\
\hline Examen general de orina & 0,110 & 70,00 & 7,71 \\
\hline Tiempo de protrombina & 0,094 & 30,00 & 2,82 \\
\hline \multicolumn{4}{|l|}{ Estudios de imagen } \\
\hline Magnificación & 0,008 & 300,00 & 2,45 \\
\hline Tele de tórax & 0,057 & 400,34 & 22,88 \\
\hline Electrocardiograma & 0,057 & 265,61 & 15,18 \\
\hline Total & & & 852,46 \\
\hline
\end{tabular}

${ }^{*}$ Pesos mexicanos

Tabla III

PROYECCIONES DEL COSTO DEL PROCESO DIAGNÓSTICO DE CÁNCER DE MAMA

\begin{tabular}{cc}
\hline Población & Costo total $^{\star}$ \\
\hline 1 & 852,46 \\
100 & $85.246,00$ \\
1000 & $852.459,97$ \\
10000 & $8.524 .599,70$ \\
100000 & $85.245 .997,03$ \\
1000000 & $852.459 .970,26$
\end{tabular}

${ }^{*}$ Pesos mexicanos

\section{DISCUSIÓN}

El cáncer de mama y el proceso diagnóstico se constituye como un serio problema de salud pública por las consecuencias fatales y la cantidad de recursos requeridos para su diagnóstico y tratamiento. En la actualidad a nivel mundial se cuenta con programas de detección oportuna de cáncer de mama, no obstante se observa una alta incidencia en etapas avanzadas $(10,11)$.

En este contexto la importancia del estudio que aquí se presenta, radica en que el proceso diagnóstico para el cáncer de mama corresponde a una política de salud que requiere de planeación y asignación de recursos, la cual desde el punto de vista 


\section{Tabla IV}

\section{COSTO POR ESCENARIO DIAGNÓSTICO DEL PROCESO DIAGNÓSTICO DEL CÁNCER DE MAMA}

\begin{tabular}{|c|c|c|c|}
\hline \multicolumn{4}{|c|}{ Escenario diagnóstico } \\
\hline Intervenciones & Uso & Costo unitario & Costo total \\
\hline \multicolumn{4}{|c|}{ A partir de medicina preventiva } \\
\hline Medicina preventiva & 1 & 95,57 & 95,57 \\
\hline Mastografía & 1 & 368,95 & 368,95 \\
\hline Consulta de médico familiar & 1 & 133,96 & 133,96 \\
\hline Costo total & & & 598,48 \\
\hline \multicolumn{4}{|c|}{ A partir de médico familiar } \\
\hline Consulta de médico familiar & 2 & 133,96 & 267,91 \\
\hline Mastografía & 1 & 368,95 & 368,95 \\
\hline Costo total & & & 636,87 \\
\hline \multicolumn{4}{|c|}{ A partir de medicina familiar con envío a clínica de mama } \\
\hline Mastografía & 1 & 368,95 & 368,95 \\
\hline Consulta de médico familiar & 2 & 133,96 & 267,91 \\
\hline Consulta de clínica de mama & 1 & 179,04 & 179,04 \\
\hline Costo total & & & 815,90 \\
\hline \multicolumn{4}{|c|}{ A partir de clínica de mama con toma de biopsia aspiración de aguja fina } \\
\hline Mastografía & 1 & 368,95 & 368,95 \\
\hline Consulta de médico familiar & 2 & 133,96 & 267,91 \\
\hline Consulta de clínica de mama & 2 & 179,04 & 358,07 \\
\hline Biopsia & 1 & 253,32 & 253,32 \\
\hline Costo total & & & $1.248,26$ \\
\hline \multicolumn{4}{|c|}{ A partir de clínica de mama con toma de biopsia excisional } \\
\hline Mastografía & 1 & 368,95 & 368,95 \\
\hline Consulta de médico familiar & 2 & 133,96 & 267,91 \\
\hline Consulta de clínica de mama & 2 & 179,04 & 358,07 \\
\hline Biopsia & 1 & $1.176,42$ & $1.176,42$ \\
\hline Costo total & & & $2.171,35$ \\
\hline \multicolumn{4}{|c|}{ A partir de clínica de mama con toma de biopsia marcaje con arpón } \\
\hline Mastografía & 1 & 368,95 & 368,95 \\
\hline Consulta de médico familiar & 2 & 133,96 & 267,91 \\
\hline Consulta de clínica de mama & 2 & 179,04 & 358,07 \\
\hline Biopsia & 1 & $4.165,17$ & $4.165,17$ \\
\hline Costo total & & & $5.160,11$ \\
\hline
\end{tabular}

económico y clínico, han demostrado la efectividad para el diagnóstico temprano. Para el tomador de decisiones, identificar el costo de un programa de detección oportuna dirigida a población sana que se encuentra en riesgo, permite la planeación de las acciones e intervenciones que en consecuencia incrementen las probabilidades de éxito.

Desde esta perspectiva, abordar el costo promedio del proceso diagnóstico para el cáncer de mama, ponderado por el porcentaje de población sana que requiere tamizaje, y el porcentaje de población sospechosa que requiere otras acciones de salud, permite identificar el costo real para así aplicarlo a una población específica.

En la población estudiada se observa baja escolaridad, esto difiere de lo publicado en la literatura, la cual señala que a mayor escolaridad el uso de los servicios de salud es más alto (12). Una posi- 
ble explicación de esta diferencia puede ser el tipo de población abordada, la cual se encuentra muy cerca del adulto mayor, y se debe reconocer que pertenece a la generación en la cual el acceso al sistema educativo se encontraba muy limitado.

En este estudio la prevalencia de mastografía normal es superior al $80 \%$, cifra cercana a lo reportado en otros estudios $(73,0 \%$ y $92,9 \%)(13,14)$, esta similitud de los resultados estriba en que la población analizada pertenece a población sana que se encuentra en riesgo, desde esta perspectiva la prevalencia de normalidad es la esperada.

Identificar la incidencia de cáncer de mama no es el tema específico del artículo que se presenta, no obstante es digno mencionar que el valor encontrado $(2,4 \%)$ es similar a lo que reporta en México el Instituto Nacional de Estadística y Geografía (10).

El costo unitario más alto corresponde a la biopsia, no obstante por la propuesta del artículo la estimación del costo promedio se disminuye notablemente, ya que el costo original se pondera en función del uso promedio del servicio de toda la población estudiada. Este es un abordaje que contempla la perspectiva del sistema de salud, en la cual la unidad de análisis es la población, para posteriormente poder extrapolar la información al individuo. Bajo esta misma lógica tendría que explicarse el costo del proceso diagnóstico del cáncer de mama el cual aparentemente es bajo. La diferencia se acentúa cuando se compara con cifras publicadas en la literatura las cuales señalan costos de US\$2.450 y US\$34.600 por año de vida ganado (15).

Cuando se aborda el proceso diagnóstico del cáncer de mama se debe tener claro que las acciones de salud difieren en población sana y población sospechosa. Al respecto se puede decir que en población sana las acciones de salud concretas para realizar el diagnóstico temprano incluyen educación, autoexploración, examen clínico de mama y la mastografía (3). Cuando el proceso diagnóstico se aplica en población sospechosa los métodos contemplan ecografía mamaria y la biopsia entre otros. Los métodos y acciones señalados son los incluidos en el costeo realizado en el trabajo que aquí se presenta.

Es necesario aclarar que el costo del diagnóstico de cáncer de mama difiere del costo del proceso diagnóstico. En relación a este último existen diferentes alternativas de acción que se pueden adoptar. Si se estima el costo del proceso en una paciente en la cual se confirma el diagnóstico de cáncer de mama, el costo promedio se incrementa notablemente, escenario no observado en una paciente sometida exclusivamente a una prueba de tamizaje, pero la propuesta del artículo es estimar el costo promedio en una población dada en la cual existen individuos sanos e individuos con la patología.
El costo promedio identificado $(\$ 852,46)$ en relación a los publicados en la literatura (9) es ostensiblemente menor, la explicación ya se ha discutido en esta sección del artículo y va en relación con la estimación del costo desde la perspectiva sistémica, la cual es la adecuada para hacer proyecciones de la asignación del presupuesto a este programa en particular. En consecuencia las proyecciones que se presentan en este artículo se apegan a la realidad del sistema de salud.

Cuando se analizan los diferentes escenarios del proceso diagnóstico del cáncer de mama se aprecian variaciones importantes en los costos que van desde $\$ 598,00$ a $\$ 5.160,00$. Esta información es un valor agregado del artículo que puede ser de utilidad para el directivo responsable de asignar los recursos.

\section{CONCLUSIÓN}

El costo del proceso diagnóstico del cáncer de mama en México es $\$ 852,46$. El costo del proceso diagnóstico tiene una mayor efectividad cuando se compara con el tratamiento del cáncer de mama. Por lo tanto, someter a una paciente a tamizaje ofrece mejores perspectivas para el paciente y para el sistema de salud.

\section{REFERENCIAS}

1. Knaul M, Nigenda G, Lozano R, Arreola-Órnelas $\mathrm{H}$, Langer A, Frenk J. Cáncer de mama en México: una prioridad apremiante. Salud Publica Mex 2009;51:S335-S344.

2. Maza-Fernández M, Vecchi-Martini E. El cáncer de mama en México: evolución, panorama actual y retos de la sociedad civil. Salud Publica Mex 2009;51:S329S334.

3. Secretaria de Salud. Manual de exploración clínica de las mamas; 2007. Disponible en: http://www.isea.gob. $\mathrm{mx} /$ formatos/Cancermama/CLINICAMAMAS.pdf

4. Harris JR, Lippman ME, Morrow M, Osborne CK, Enfermedades de la mama. España: Editorial Marban Libros; 2009.

5. Diagnóstico precoz del cáncer de mama en medicina familiar. Publicación de la Sociedad Andaluza de Medicina Familiar y Comunitaria. Disponible en: http:// www.fecma.org/archivosfecma/informacion/diagnostico_precoz.pdf

6. Berry A, Cronin A, Plevritis K, Fryback G, Clarke L, Zelen $\mathrm{M}$, et al. Effect of screening and adjuvant therapy on mortality from breast cancer. $\mathrm{N}$ Engl $\mathrm{J}$ Med 2005;353:1784-92.

7. Organización Panamericana de la Salud (OPS). Alcance y aplicación de la economía de la salud. Serie Desarrollo de la Representación de la OPS/OMS en Cuba, 24; 2001.

8. Marie K, Arreola-Órnelas H, Velázquez E, Dorantes $\mathrm{J}$, Ávila-Burgos L. El costo de la atención médica del 
cáncer mamario: el caso del Instituto Mexicano del Seguro Social. Salud Publica Mex 2009;51:S286S295.

9. Trillo E, Castaño F, Rubio E, Rubio E. Estudio costeefectividad de un programa de detección precoz del cáncer de mama. Rev Esp Econ Salud 2005;4:219-30.

10. Instituto Nacional de Geografía y Estadística [acceso 24 enero 2012] Disponible en: http://www.inegi.org. $\mathrm{mx} /$ inegi/contenidos/espanol/prensa/contenidos/estadisticas/2011/cancer11.asp?s=inegi\&c=2781\&ep=51.

11. Knaul F, López-Carrillo L, Lazcano-Ponce E, GómezDantés H, Romieu I, Torres G. Cáncer de mama: un reto para la sociedad y los sistemas de salud. Salud Publica Mex 2009; 51:S3138-S140.

12. López-Carrillo L, Suárez-López L, Torres-Sánchez L. Detección del cáncer de mama en México: síntesis de los resultados de la encuesta nacional de salud reproductiva. Salud Publica Mex 2009; 51:S345-S349.

13. Silva L, Ríos N. Estudio mamario integral en el Hospital General de México: frecuencia y clasificación BI-RADS. Experiencia de un año. An Radiol Mex 2011;2:91-7.

14. Jaramillo N, Mantilla J, Rey J. Hallazgos clínicos, mamográficos y ecográficos en un programa comunitario de tamizaje para detección temprana de cáncer de seno en la ciudad de Bucaramanga. Med UNAB 2007;10:28-32.

15. Brown L, Goldie J. Health service interventions for cancer control in developing countries. Chapter 29. En: Jaminson TM, Bremen G, Meashan R. Disease Control Priorities in Developing Countries. 2nd ed. New York. Oxford University Press/World Bank; 2006. 